\title{
Application of Explicitly Localized Molecular Orbitals to Electronic Structure Calculations
}

\author{
Piotr de Silva ${ }^{\aleph^{\star a b}}$, Marcin Makowski ${ }^{\star a}$, and Jacek Korchowiec ${ }^{\star a}$ \\ §SCS-Metrohm Foundation Award winner for best oral presentation
}

\begin{abstract}
We have recently generalized the method for localizing orbitals on a set of predefined molecular fragments [Phys. Chem. Chem. Phys. 2012, 14, 546]. The regional localized molecular orbitals (RLMO) are well suited for exploiting the locality of electronic correlation at post-Hartree-Fock level of theory. In this paper, the adequacy of RLMO representation is tested in the second-order local Møller-Plesset (LMP2) perturbation theory. Two model systems, namely, $n$-pentadecane and trans-retinal, are considered. Adequacy of RLMO/LMP2 method is discussed in conjunction with 'exact' MP2 and Pipek-Mezey LMP2 calculations. It is demonstrated that RLMO/MP2 method reduces correlation space and reproduces more than $99 \%$ of the correlation energy.
\end{abstract}

Keywords: Correlation energy - Local Møller-Plesset second-order perturbation theory (local MP2) .

Molecular orbital localization schemes · Regional localized molecular orbitals (RLMO)

\section{Introduction}

Standard first-principle quantumchemical calculations are based on canonical molecular orbitals (CMO) that extend over the whole molecule. The lack of locality in CMO representation is responsible for an unfavourable scaling of the electronic structure calculations with the system's size. Molecular orbitals (MO) are only auxiliary one-electron functions and any unitary transformation of them preserves the total wavefunction. Therefore, the works are carried out in quest of the best local molecular orbital (LMO) representation. ${ }^{[1-14]}$ Such a representation should utilize the locality and should relate directly most traditional chemical concepts.

There is no unique choice of a unitary transformation to get localized orbitals and several methods have been proposed in the literature. The best known schemes

\footnotetext{
${ }^{\star}$ Correspondence: P. de Silva ${ }^{\mathrm{ab}}$, M. Makowski ${ }^{\mathrm{a}}$, J. Korchowiec ${ }^{a}$

Tel.: +48 12663 2026;

E-mail: desilva@chemia.uj.edu.pl

Tel.: +48 126632028 ;

E-mail: makowskm@chemia.uj.edu.pl

Tel: : +48 126632030 ;

E-mail: korchow@chemia.uj.edu.pl

aK. Gumiński Department of Theoretical Chemistry

Faculty of Chemistry, Jagiellonian University

R. Ingardena 3, 30-060 Kraków, Poland

'Département de Chimie Physique

Université de Genève

30, quai Ernest-Ansermet

$\mathrm{CH}-1211$ Genève 4
}

are these proposed by Boys, ${ }^{[1,2]}$ EdmistonRuedenberg[3] and Pipek-Mezey. ${ }^{[8]}$ They are based on different localization criterion, but the resulting orbitals are similar and agree with chemical intuition. In the Boys localization procedure ${ }^{[1]}$ the spatial extent of orbitals is minimized. The EdmistonRuedenberg localization scheme ${ }^{[3]}$ maximizes the electronic self-repulsion energy of the orbitals. Pipek-Mezey method ${ }^{[8]}$ maximizes the sum of squares of Mulliken atomic populations. These schemes are based on an iterative procedure relying on consecutive $2 \times 2$ Jacobi rotations up to convergence, which is very slow especially for huge systems and virtual subspaces for extended basis sets.

Localization in the virtual MO subspace is of crucial importance for performance of fragmentation computational methods at Hartree-Fock (HF), ${ }^{[15-17]}$ Kohn-Sham (KS) ${ }^{[18]}$ and post $-\mathrm{HF}^{[19]}$ levels of theory. Subotnik et al. ${ }^{[10]}$ proposed a method where the atomic orbital (AO) space is partitioned into minimal basis and hard virtuals subspaces. Jansík et al. ${ }^{[12]}$ have generalized the Boys method ${ }^{[1]}$ by minimizing powers of orbital variance. They used a trust-region algorithm instead of Jacobi sweeps, which enables localization of virtual orbitals. Other localization schemes originate from one-particle density matrices. Reed and Weinhold have proposed natural localized molecular orbitals (NLMO) ${ }^{[7]}$ that arise from diagonalization of the density matrix with ensured minimal mixing between strongly and weakly occupied natural bond orbitals (NBO). ${ }^{[6]}$ Cholesky decomposition of the density matrix was proposed by Aquilante et al.[11]

We have recently generalized the regional localized molecular orbital method ${ }^{[9]}$ into a multi-fragment formulation. ${ }^{[14]}$ The procedure was efficient and worked well in both occupied and virtual subspaces. It was argued that RLMO are well suited for post-Hartree-Fock (post-HF) calculations. Here we check its performance in local second-order Møller-Plesset (LMP2) perturbation theory. The paper is organized as follows. First, we describe the RLMO method. Then the LMP2 formalism is discussed. After a short section on computational details, the preliminary results are reported and, finally, the conclusions are given along with closing remarks.

\section{Regional Localized Molecular Orbitals}

RLMOs are obtained directly from the density matrix $(\boldsymbol{D}),{ }^{[9,14]}$ so no initial orbitals are needed. The main idea of the RLMO localization procedure is to find a set of eigenvectors of $\boldsymbol{D}$ that resembles fragment molecular orbitals as much as possible. In principle, there is an infinite number of different sets of eigenvectors since eigenvalues of $\boldsymbol{D}$ are extremely degenerate. There are only two possible eigenvalues at RHF level of theory. Occupied molecular orbitals have eigenvalues (occupation numbers) equal to 2 , while virtual orbitals have ei- 
genvalues equal to 0 . Each rotation of MOs within the occupied or virtual subspace gives another set of eigenvectors. CMOs are of special importance since in addition they diagonalize the Fock matrix.

The RLMO localization procedure was described elsewhere. ${ }^{[9,14]}$ Here, we give a short sketch of the localization scheme. First, the density matrix is transformed to the orthogonalized $\mathrm{AO}$ basis:

$$
D^{\mathrm{OAO}}=\boldsymbol{S}^{1 / 2} \boldsymbol{D} \boldsymbol{S}^{1 / 2}
$$

where $S$ is the overlap matrix. Next, the regional orbital (RO) space is constructed by the diagonalization of diagonal blocks $\left(\boldsymbol{D}_{i}^{\mathrm{OAO}}\right)$ of $\boldsymbol{D}^{\mathrm{OAO}}$ associated with predefined molecular fragments:

$$
\boldsymbol{D}_{i}^{\mathrm{RO}}=\boldsymbol{T}_{i}^{\dagger} \boldsymbol{D}_{i}^{\mathrm{OAO}} \boldsymbol{T}_{i}
$$

where $\boldsymbol{T}_{i}$ collects eigenvectors of $i$-th molecular fragment. Their eigenvalues, diagonal elements of $\boldsymbol{D}_{i}^{\mathrm{RO}}$, fall into one of the three types: approximately doubly occupied, singly occupied and empty ROs. A singly occupied regional orbital appears only if a covalent bond between fragments is cut. In this case a hybrid type orbital is obtained. Hybrid orbitals have partners in the neighbouring fragments. The full transformation matrix from the OAO to RO basis set is given by the direct sum:

$$
\mathbf{T}=\underset{i}{\oplus} \mathbf{T}_{i}
$$
basis,

Finally, the density matrix in the RO

$$
\boldsymbol{D}^{\mathrm{RO}}=\boldsymbol{T}^{\dagger} \boldsymbol{D}^{\mathrm{OAO}} \boldsymbol{T}
$$

is diagonalized:

$$
\boldsymbol{D}^{\mathrm{RLMO}}=\boldsymbol{U}^{\dagger} \boldsymbol{D}^{\mathrm{RO}} \boldsymbol{U}
$$

This transformation restores the exact occupation numbers, therefore the diagonal elements of $\boldsymbol{D}^{\mathrm{RLMO}}$ are equal to 2 or 0 . Back transformation :

$$
\boldsymbol{C}_{A O}^{R L M O}=\boldsymbol{S}^{-1 / 2} \boldsymbol{T U}
$$

gives the resultant RLMO. These orbitals should resemble those of the RO representation.

\section{Local MP2 Formalism}

The electron correlation energy can be approximated by the following secondorder Møller-Plesset (MP2) correction:

$$
E_{2}=\sum_{i}^{o c c} \sum_{j} \sum_{a} \sum_{b}^{\text {virt virt }} \frac{(i a \mid j b)(2(i a \mid j b)-(i b \mid j a))}{\varepsilon_{i}+\varepsilon_{j}-\varepsilon_{a}-\varepsilon_{b}} \text { (7) }
$$

where $\varepsilon_{i}$, $\varepsilon_{j}$ and $\varepsilon_{a}, \varepsilon_{b}$ are energies of occupied and virtual CMOs, respectively.
Two-electron integrals, (ia $\mid j b$ ), are written in the chemical notation. This is the main direct contribution to the correlation energy for closed-shell systems arising from double excited configurations. Other excitations are excluded either due to the Slater-Condon rules or due to Brillouin theorem and can contribute to the correlation energy by high order corrections. One should remember that this very simple formula is valid only in the CMO basis. Unfortunately, it also suffers from the delocalized nature of CMOs. Namely, the number of significant two-electron integrals scales as $O\left(N^{4}\right)$ and transformation of integrals from the AO to the CMO basis scales as $O\left(N^{5}\right)$. Such scaling properties are highly unfavorable and make the treatment of large systems practically impossible (CPU and memory usage). On the other hand, the correlation in isolators is a very local effect decaying with electron distance as $r^{-6}$. This leads at once to the conclusion that high cost of standard MP2 technique results from the CMO basis set.

The Local MP2 (LMP2) was introduced by Saeb $\varnothing$ and Pulay. ${ }^{[20]}$ The use of LMO basis exploits locality of the correlation effect. The formalism is based on Hylleraas functional:

$$
\left\langle 1\left|\hat{H}_{0}-E_{0}\right| 1\right\rangle+2\left\langle 1\left|\hat{H}_{1}-E_{1}\right| 0\right\rangle \geq E_{2}
$$

that provides a variational upper bound to $E_{2}$. Here, $|0\rangle$ is the RHF wave function, $|1\rangle$ is the first order correction to $|0\rangle$. Unperturbed Hamiltonian $\hat{H}_{0}$ is a sum of Fock operators. The correlation (fluctuation) potential $\hat{H}_{1}$ is a difference between exact non-relativistic Hamiltonian and $\hat{H}_{0}$. Eqn. (8) can be used to derive the main working equations of LMP2. Here, we briefly review LMP2 procedure as described in ref. [19]. Let us assume that:

$$
|1\rangle=\frac{1}{2} \sum_{i j \in P} \sum_{a b \in[j]} T_{a b}^{i j}\left|0^{i j \rightarrow a b}\right\rangle
$$

where $T_{a b}^{i j}$ is an amplitude of $i j \rightarrow a b$ double excitation and $\left|0^{i j_{\rightarrow} a b}\right\rangle$ is a double excited configuration. By $P$ we mean the set of significant occupied pairs, i.e. the pairs for which correlation effects are considered. The symbol $[i j]$ denotes the virtual orbital domain spanned for the $i$-th and $j$-th orbitals. By inserting the expansion (9) into inequality (8) the following set of equations can be derived:

$$
\boldsymbol{0}=\boldsymbol{K}^{i j}+\boldsymbol{F} \boldsymbol{T}^{i j}+\boldsymbol{T}^{i j} \boldsymbol{F}+\sum_{k} F_{i k} \boldsymbol{T}^{k j}+\boldsymbol{T}^{i k} F_{k j}
$$

where $\boldsymbol{K}^{i j}$ and $\boldsymbol{T}^{i j}$ are matrices indexed by virtual orbital pairs belonging to $[i j]$ and $\boldsymbol{F}$ is the corresponding virtual-virtual block of the Fock matrix. The matrix $\boldsymbol{K}^{i j}$ groups two-electron integrals $\left[K_{a b}^{i j}=(i a \mid j b)\right]$ of the occupied $(i, j)$-pair with all virtual $(a$, $b)$-pairs from $[i j]$. There are as many equa- tions of type (10) as there are $(i, j)$-pairs in the $P$ set. The equations are coupled through the nondiagonal Fock matrix elements, therefore they have to be solved iteratively. Our approach is simpler than the original LMP2 formulation since virtual domains are built from orthonormal LMOs instead of redundant projected AOs. ${ }^{[20]}$ After reaching convergence $E_{2}$ is calculated from the following equation:

$$
E_{2}=\sum_{i j \in P} \sum_{a b \in i j]}\left(2 T_{a b}^{i j}-T_{b a}^{i j}\right) K_{a b}^{i j}
$$

The formalism is exact and is valid for each molecular orbital basis sets, including CMO one. As long as all occupied and virtual pairs are taken into account, the energies computed from Eqns. (7) and (11) are identical within assumed accuracy.

The computational costs can be lowered by considering the locality of correlation effects. Namely, the correlation for $(i, j)$-pair is significant if both orbitals are spatially near to each other. The virtual orbitals have to be added to [ij], if they are spatially near to $(i, j)$-pair. In the asymptotic limit the number of significant $(i, j)$-pairs should grow linearly with the system's size. In addition, the size of each virtual domain is almost the same, therefore, the LMP2 formalism should show linear scaling behavior.

\section{Model Systems and Computational Results}

We have chosen two model systems, namely $n$-pentadecane and trans-retinal, to test the adequacy of LMP2 formalism in the RLMO basis set. The first system is taken in all-trans conformation and has only $\sigma$-bonds. The second molecule possesses in addition a system of coupled $\pi$-bonds and one six-member ring. The structure of both molecules is shown in Fig. 1. The broken lines indicate how the molecules were divided into fragments. There are 15 fragments in the $n$-pentadecane molecule. Each fragment encloses a carbon atom and its adjacent hydrogen atoms. The second molecule was divided into 13 fragments. The system was never cut through double bonds since we prefer to preserve $\sigma / \pi$ separation.

Structures of both molecules were optimized at HF/6-31G(d) level of theory using the GAMESS package.[21] The RLMO localization and LMP2 schemes were implemented in the GAMESS package. Degree of localization is described by the normalized partial norm, $L_{i}^{j},{ }^{[14]}$ that measures $i$-th orbital's projection on $j$-th fragment's $\mathrm{AO}$ basis. Of course, the $i$-th orbital is associated with the $j$-th fragment and normalization means that $\sum_{j} L_{i}^{j}=1$. Depending on $L_{i}^{j}$, RLMOs (possibly CMOs) are classified 


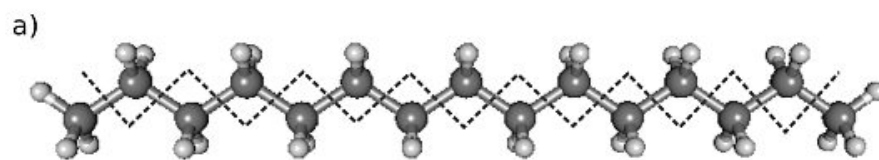

b)

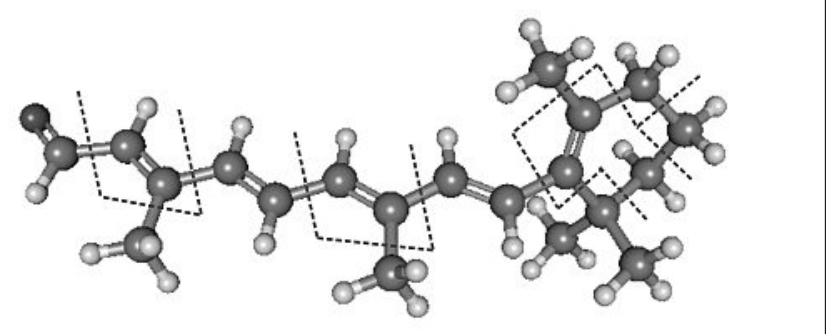

as: (1) very well localized, $L_{i}^{j} \in[0.99,1]$; (2) well localized, $L_{i}^{j} \in[0.90,0.99)$; (3) moderately localized, $L_{i}^{j} \in[0.75,0.90) ;$ (4) significantly delocalized, $L_{i}^{j} \in[0.50,0.75)$; (5) delocalized, $L_{i}^{j} \in[0,0.50)$.

The choice of the correlation space is crucial for LMP2 performance. The following procedure was adopted. First we computed the relative Mulliken orbital populations. Atoms with relative population in $i$-th LMO, $\mathrm{A}_{i}>0.02$ were considered in the selection criterion. All occupied pairs with the distance between $\mathrm{A}_{i}$ and $\mathrm{A}_{i}$ centers shorter than 8 a.u. $\left[\operatorname{dist}\left(\mathrm{A}_{i}, \mathrm{~A}_{j}\right)<8\right.$ ] were included in $P$. The virtual domains were constructed in slightly different manner. Eqn. (10) was solved for diagonal occupied pairs [(ii)-pairs]. The virtual orbital $a$ belonged to $[i i]$ only if the absolute value of $T_{a a}^{i i}$ was greater than the threshold value (0.0001). Virtual domain [ij] includes all virtual orbitals from the $[i i]$ and $[j j]$ domains. More information how to construct $P$ set and its virtual domains are given in our previous paper. ${ }^{[19]}$

\section{Results and Discussion}

The quality of localization of RLMOs is shown in Fig. 2. The upper panel corresponds to pentadecane. Pentadecane is a covalently bonded system. The molecule is partitioned into fifteen fragments. All single $\mathrm{C}-\mathrm{C}$ covalent bonds are broken by this division. These bonds are restored during the localization procedure and have practically a two-center nature. The $\mathrm{C}-\mathrm{C}$ bonding orbitals distinguish additional bonding regions between predefined fragments. Their degree of localization is the sum of two highest $L_{i}^{j}$ coefficients. It can be seen from the histogram $2 \mathrm{a}$ that occupied RLMOs (dark gray bars) are at least well localized. Their degree of localization $L_{i}^{j}$ is always greater than 0.90 . As one should expect, localization in the virtual subspace is worse than in the occupied one. Now, $80 \%$ of virtual RLMOs are well or very well localized $\left(L_{i}^{j} \geq 0.90\right)$. Remaining orbitals constitute moderately localized
Fig. 1. Conformation of $n$-pentadecane (a) and trans-retinal (b).

The broken lines separate the molecules into predefined molecular fragments.
$\left(L_{i}^{j} \in[0.75,0.90)\right)$ and significantly delocalized $\left(L_{i}^{j} \in[0.50,0.75)\right)$ categories of orbitals. We have not plotted the histograms showing the quality of localization of CMOs as all virtual and almost $95 \%$ of occupied orbitals are delocalized $\left(L_{i}^{j}<0.50\right)$. Histogram $2 \mathrm{~b}$ illustrates the quality of localization for the trans-retinal molecule. The system of five $\pi$-coupled orbitals and the six-member ring are responsible for a slightly worse localization as compared to $n$-pentadecane. Now, delocalized orbitals $\left(L_{i}^{j}<0.50\right)$ are present among occupied RLMOs. Nevertheless, more than $88 \%$ or $84 \%$ of occupied or virtual RLMOs have $L_{i}^{j}>0.90$. Except for a few core orbitals, a)

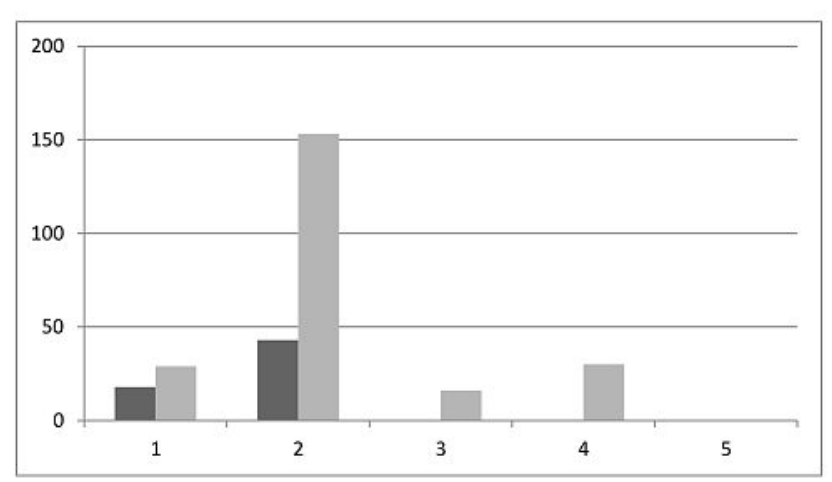

b)

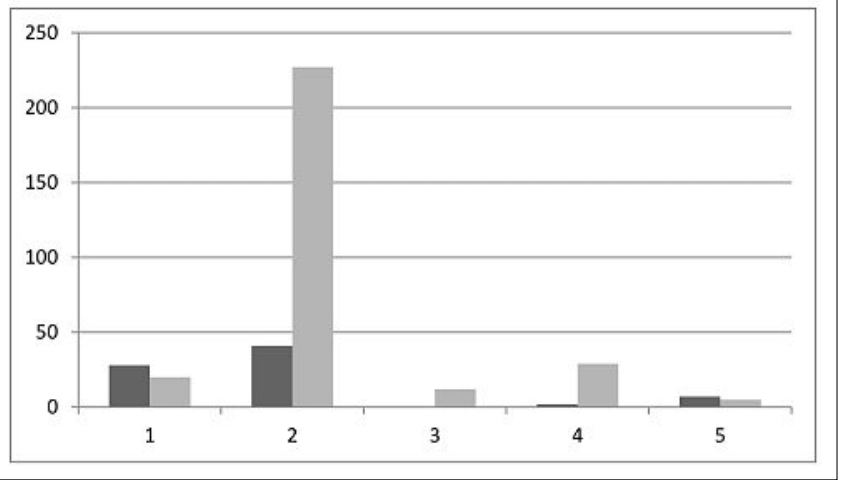

a)

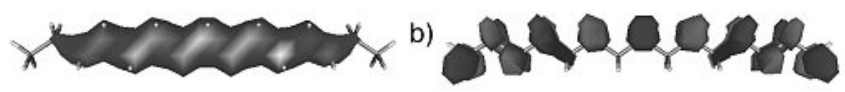

c)

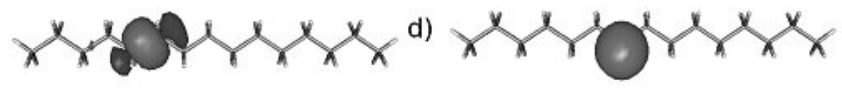

occupied CMOs are completely delocalized. Almost $90 \%$ of virtual CMO in trans-

The shape of selected occupied CMOs and RLMOs for $n$-pentadecane is shown in Fig. 3. Panels a and $b$ correspond to $\mathrm{C}-\mathrm{C}$ and $\mathrm{C}-\mathrm{H}$ orbitals, respectively. The orbitals are completely delocalized and almost all fragments are involved in their formation. Panels c and d illustrate occupied RLMOs. They correspond to the $\mathrm{C}-\mathrm{C}$ bonding region and $\mathrm{CH}_{2}$ fragment. Due to RLMO construction the latter orbitals have local $\mathrm{C}_{2 \mathrm{y}}$ symmetry (approximate symmetry of diagonal blocks of $\boldsymbol{D}^{\mathrm{OAO}}$ ). Terminal $\mathrm{CH}_{3}$ groups have local $\mathrm{C}_{3 \mathrm{v}}$ symmetry. As long as coupling between ROs is weak this symmetry is preserved. Notice that final diagonalization is performed in RO representation. Each RO has a well-defined local symmetry. Therefore, the shape of these orbitals can be obtained from elementary considerations within a given point group symmetry $\left(a_{1}\right.$ and $b_{1}$ for $C_{2 v}$ and $\mathrm{a}_{1}$ and $\mathrm{e}$ for $\mathrm{C}_{3 \mathrm{v}}$ local symmetries). The electronic structure of the whole molecule is composed of such orbitals supplemented by core orbitals. Of course, none of the RLMOs has a proper system's symmetry $\left(\mathrm{C}_{2 \mathrm{v}}\right)$. The symmetry of $\mathrm{C}_{15} \mathrm{H}_{32}$ (all-trans configuration) caused that core $\mathrm{CMOs}$ are delocalized. retinal are delocalized.
Fig. 2. Degrees of localization in RLMO scheme for $n$-pentadecane (a) and trans-retinal (b). Entries on horizontal axes correspond to 1: very well localized, 2: well localized, 3 : moderately localized, 4: significantly delocalized, 5: delocalized RLMOs. Occupied and virtual RLMOs are distinguished by dark and light gray bars, respectively.
Fig. 3. Occupied CMOs $(a, b)$ and RLMOs (c,d) representing $\mathrm{C}-\mathrm{C}$ and $\mathrm{C}-\mathrm{H}$ bonding patterns in $n$-pentadecane (isovalue $=0.025$ ) . 
Two selected CMOs and two selected RLMOs for trans-retinal are shown in Fig. 4. Panels $\mathrm{a}$ and $\mathrm{b}$ correspond to occupied $\sigma$ and $\pi$ CMOs. The worst localized $\sigma$ and $\pi$ RLMOs are drawn in panels c and d, respectively. CMOs are completely delocalized. In contrast, delocalized $\pi$-RLMOs are spread over four fragments and this manifests the presence of coupled $\pi$-bonds in a system. The worst localized are RLMOs assigned to fragments belonging to the ring but even here, a great number of RLMOs is quite well localized.

The performance of RLMO/LMP2 using 6-31G(d) basis set for both systems is summarized in Table 1. For comparative purposes we have also performed standard MP2 calculation and LMP2 with PipekMezey (P-M) localized MO. ${ }^{[8]}$ The number of important pairs $n_{i j}$ in RLMO/LMP2 is halved as compared to CMO calculations and slightly higher as compared to $\mathrm{P}-\mathrm{M}$ calculations. All $\mathrm{C}-\mathrm{H}$ bonds in $\mathrm{P}-\mathrm{M}$ scheme are centered on two atoms only. In contrast RLMOs of $\mathrm{CH}_{2}$ fragments are three-center orbitals while those of terminal $\mathrm{CH}_{3}$ fragments are four-center orbitals. This explains the increase in the number of important pairs. Total number of $\mathrm{ij} \rightarrow \mathrm{ab}$ excitation is only slightly higher at RLMO/ LMP2 than at P-M/LMP2 level as compared to standard MP2 calculations. The same cutoff criteria used in RLMO/MP2 calculations allow to restore more correlation energy than in M-P/LMP2 calculations. One should expect such behavior since RLMOs are more spread in space than P-M LMOs. Both sets of LMOs reproduce more than $99 \%$ of the correlation energy. For the second system differences are more pronounced. The size of molecular fragments for the trans-retinal molecule is bigger than for the $n$-pentadecane molecule. Therefore, the difference in the number of important occupied pairs is more pronounced. The number of double excited configurations $n_{i j \rightarrow a b}$, at the RLMO/ LMP2 level of theory is four times lower than for conventional MP2 calculations. This ratio is equal to 5.8 for $\mathrm{P}-\mathrm{M} / \mathrm{LMP} 2$ calculations. Again RLMO reference basis restores more correlation energy than P-M LMO basis.

The analyzed molecular systems are relatively small. The advantage of RLMO basis will be more pronounced for bigger systems since P-M localization scheme is very slow in the virtual subspace. For the $n$-pentadecane (trans-retinal) molecule, RLMO procedure is eleven (twelve) times faster than the P-M scheme.

\section{Conclusions}

In this paper we have applied RLMOs to LMP2 calculations. LMP2 takes the advantage of the localization to reduce the number of important double excited configurations. The efficiency of RLMO/ LMP2 electronic structure calculations on model systems is compared to standard MP2 and P-M/LMP2 calculations. RLMO basis reproduces more correlation energy a)

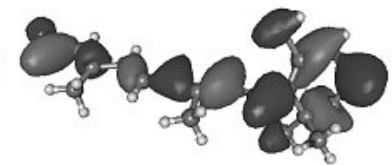

c)

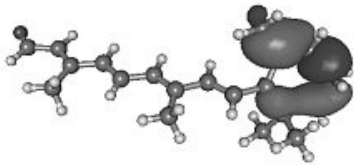

b)

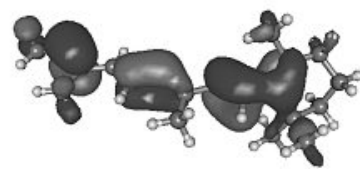

d)

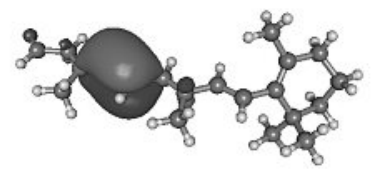

Fig. 4. Occupied CMOs $(a, b)$ and RLMOs (c,d) representing $\sigma$ and $\pi$ bonding patterns in trans-retinal (isovalue $=0.025)$. RLMOs are from the delocalized domain (see the caption under Fig. 2b).

Table 1. The main characteristics of MP2 and LMP2 calculations: number of important pairs $\left(n_{i j}\right)$, size of the correlation space $\left(n_{i j \rightarrow a b}\right)$, second-order correction (MP2 correlation energy) to the energy $\left(E_{2}\right)$ and percentage of the restored correlation energy. The first and the second entries correspond to $n$-pentadecane and trans-retinal, respectively.

\begin{tabular}{|c|c|c|c|}
\hline & CMO basis & RLMO basis & P-M basis \\
\hline & \multicolumn{3}{|c|}{$n$-pentadecane } \\
\hline$n_{i j}$ & 1891 & 961 & 851 \\
\hline$n_{i j \rightarrow a b}$ & 24683223 & 4471358 & 4295560 \\
\hline$E_{2}[$ a.u. $]$ & -2.03958 & -2.02973 & -2.02590 \\
\hline \multirow[t]{2}{*}{$\%$ of correlation } & 100 & 99.52 & 99.32 \\
\hline & \multicolumn{3}{|c|}{ trans-retinal } \\
\hline$n_{i j}$ & 3081 & 1753 & 1512 \\
\hline$n_{i j \rightarrow a b}$ & 66350875 & 16343339 & 11415407 \\
\hline$E_{2}[$ a.u. $]$ & -2.85268 & -2.83151 & -2.82025 \\
\hline$\%$ of correlation & 100 & 99.26 & 98.86 \\
\hline
\end{tabular}

than P-M basis for the same cutoff criteria used to construct the correlation space. It is connected with spatial spreads of RLMOs. For a $\sigma$-bonded system the correlation space is only slightly larger than within P-M/LMP2 approach. In a $\pi$-conjugated system differences are more pronounced. Nevertheless, the correlation space is much smaller than in the case when CMOs are used. The results obtained indicate that RLMOs can be applied at LMP2 level of theory for bigger systems. Such calculations are expected to give significant CPU and memory savings.

\section{Acknowledgements}

This work was supported by the International $\mathrm{PhD}$ studies programme at the Faculty of Chemistry, Jagiellonian University within the Foundation for Polish Science MPD Programme co-financed by the EU European Regional Development Fund. The calculations were carried out with the equipment purchased thanks to the financial support of the European Regional Development Fund in the framework of the Polish Innovation Economy Operational Program (contract no. POIG.02.01.00-12-023/08).

Received: January 18, 2012

[1] S. F. Boys, Rev. Mod. Phys. 1960, 32, 296.

[2] J. M. Foster, S. F. Boys, Rev. Mod. Phys. 1960 32, 300 .

[3] C. Edmiston, K. Ruedenberg, Rev. Mod. Phys. 1963, 35, 457 .

[4] V. Magnasco, A. Perico, J. Chem. Phys. 1967, 47, 971

[5] W. von Niessen, J. Chem. Phys. 1972, 56, 4290

[6] A. E. Reed, F. Weinhold, J. Chem. Phys. 1983, $78,4066$.

[7] A. E. Reed, F. Weinhold, J. Chem. Phys. 1985 83, 1736.

[8] J. Pipek, P. G. Mezey, J. Chem. Phys. 1989, 90, 4916.

[9] F. L. Gu, Y. Aoki, J. Korchowiec, A. Imamura B. Kirtman, J. Chem. Phys. 2004, 121, 10385.

[10] J. E. Subotnik, A. D. Dutoi, M. Head-Gordon, J. Chem. Phys. 2005, 123, 114108.

[11] F. Auqilante, T. B. Pedersen, A. S. de Merás, H. Koch, J. Chem. Phys. 2006, 125, 174101.

[12] B. Jansík, S. Høst, K. Kristensen, P. Jørgensen, J. Chem. Phys. 2011, 134, 194104.

[13] F. Wu, W. Liu, Y. Zhang, Z. Li, J. Chem. Theory Comput. 2011, 7, 3643.

[14] P. de Silva, M. Giebultowski, J. Korchowiec, Phys. Chem. Chem. Phys. 2012, 14, 546.

[15] J. Korchowiec, F. L. Gu, A. Imamura, B. Kirtman, Y. Aoki, Int. J. Quantum Chem. 2005 , $102,785$.

[16] M. Makowski, J. Korchowiec, F. L. Gu, Y. Aoki, J. Comput. Chem. 2006, 27, 1603.

[17] J. Korchowiec, J. Lewandowski, M. Makowski, F. L. Gu, Y. Aoki, J. Comput. Chem. 2009, 30, 2515.

[18] J. Korchowiec, P. de Silva, M. Makowski, F. L. Gu, Y. Aoki, Int. J. Quantum Chem. 2010, 110 2130.

[19] M. Makowski, J. Korchowiec, F. L. Gu, Y. Aoki, J. Comput. Chem. 2010, 31, 1733.

[20] S. Saebø, P. Pulay, Аnnu. Rev. Phys. Chem. 1993, 44, 213.

[21] M. W. Schmidt, K. K. Baldridge, J. A. Boatz, S. T. Elbert, M. S. Gordon, J. H. Jensen, S. Koseki, N. Matsunaga, K. A. Nguyen, S. Su, T. L. Windus, M. Dupuis, J. A. Montgomery, J. Comput. Chem. 1993, 14, 1347. 